\title{
PRIME AND SEMIPRIME SEMIGROUP RINGS OF CANCELLATIVE SEMIGROUPS
}

\author{
by JAN OKNIŃSKI $\dagger$
}

(Received 19 February, 1991; revised 3 September, 1991)

Introduction. Let $S$ be a cancellative semigroup. This paper is motivated by the problem of finding a description of semigroup rings $K[S]$ over a field $K$ that are semiprime or prime. Results of this type are well-known in the case of a group ring $K[G]$, cf. [8]. The description, as well as the proofs, involve the FC-centre of $G$ defined as the subset of all elements with finitely many conjugates in $G$. In [4], [5] Krempa extended the FC-centre techniques to the case of an arbitrary cancellative semigroup $S$. He defined a subsemigroup $\Delta(S)$ of $S$ which coincides with the FC-centre in the case of groups, and can be used to describe the centre and to study special elements of $K[S]$. His results were strengthened by the author in [7], where $\Delta(S)$ was also applied in the context of prime and semiprime algebras $K[S]$. However, $\Delta(S)$ itself is not sufficient to characterize semigroup rings of this type. We note that in [2], [3] Dauns developed a similar idea for a study of the centre of semigroup rings and certain of their generalizations.

In the present paper, we introduce a congruence $\omega$ on $S$ that plays the role played by the FC-centre in the class of groups. This allows us to find necessary conditions for the ring $K[S]$ to be semiprime or prime. When restricted to the class of group rings or semigroup rings of semigroups with a group of fractions, these conditions are equivalent to those characterizing semiprimeness and primeness of $K[S]$, see [7], [8]. The second main result of the paper establishes semiprimeness of $K[S]$ for every cancellative semigroup $S$ in the case where $\operatorname{ch}(K)=0$.

If $\delta$ is a congruence on a semigroup $S$, then by $I(\delta)=I_{K|S|}(\delta)$ we denote the ideal of the algebra $K[S]$ generated by the set $\{s-t \mid(s, t) \in \delta\} . B(K[S])$ stands for the prime radical of $K[S]$. If $A$ is a subset of $S$, then $\langle A\rangle$ denotes the subsemigroup of $S$ generated by $A$. If $S$ has no identity element, $S^{1}$ stands for the monoid obtained by adjoining an identity to $S$. Otherwise, we put $S^{1}=S$.

1. Reversive congruence and the sufficient conditions. For an arbitrary (not necessarily cancellative) semigroup $S$ consider the relation $\rho_{S}$ on $S$ defined by

$$
(s, t) \in \rho_{S} \quad \text { if for every } x \in S^{1} \quad s x S \cap t x S \neq \varnothing
$$

We will write $\rho$ in place of $\rho_{S}$ if unambiguous. If $x \in S^{1}$ and $(s, t) \in \rho,(t, u) \in \rho$, then $s x g=t x h$ and $t(x h) e=u(x h) f$ for some $e, f, g, h \in S$. Thus sxge $=u x h f$, which shows that $(s, u) \in \rho$. It is clear that $\rho$ is reflexive and symmetric. Moreover, $(s, t) \in \rho$ easily implies that $(s z, t z) \in \rho$ and $(z s, z t) \in \rho$ for every $z \in S$. Therefore $\rho$ is a congruence on $S$. Note that $\rho=S \times S$ whenever $S$ is a cancellative semigroup that satisfies the right Ore condition.

Let $\rho^{\prime}=\rho_{S}^{\prime}$ be the left-right dual congruence to $\rho$ and let $\tau=\tau_{S}=\rho \cap \rho^{\prime}$.

Lemma 1. Let $S$ be an arbitrary semigroup. Then

i) $\rho, \rho^{\prime}$ and $\tau$ are congruences on $S$.

ii) Let $\sigma \in\left\{\rho, \rho^{\prime}, \tau\right\}$. Then $\sigma_{S / \sigma}$ is the trivial congruence on $S / \sigma$.

iii) If $S$ is left cancellative, then $\rho$ is a left cancellative congruence on $S$.

† Supported by Polish Scientific Grant No 211179101

Glasgow Math. J. 35 (1993) 1-12. 
Proof. i) was established above.

ii) Assume that $\left(s_{\rho}, t_{\rho}\right) \in \rho_{S / \rho}$ for some $s, t \in S$, where $y_{\rho}$ denotes the image of $y \in S$ in $S / \rho$. Then, for every $x \in S^{1}$ we have $s_{\rho} x_{\rho}(S / \rho) \cap t_{\rho} x_{\rho}(S / \rho) \neq \varnothing$. It follows that $(s x u, t x z) \in \rho$ for some $u, z \in S$. Then $s x u S \cap t x z S \neq \varnothing$, so that $s x S \cap t x S \neq \varnothing$. Hence $(s, t) \in \rho$. A symmetric argument works for $\sigma=\rho^{\prime}$.

If $\left(s_{\tau}, t_{\tau}\right) \in \tau_{S / \tau}$ where $s_{\tau}, t_{\tau}$ are the images of $s, t$ in $S / \tau$, then $\left(s_{\rho}, t_{\rho}\right) \in \tau_{S / \rho}$ because the natural homomorphism $S \rightarrow S / \rho$ factors as $S \rightarrow S / \tau \rightarrow S / \rho$ (note that for every epimorphism $\phi: U \rightarrow T$ we have $(\phi(x), \phi(y)) \in \tau_{T}$ whenever $\left.(x, y) \in \tau_{U}, x, y \in U\right)$. As shown above, this implies that $(s, t) \in \rho$. Similarly $s_{\rho^{\prime}}=t_{\rho^{\prime}}$. Therefore $s_{\tau}=t_{\tau}$, which proves ii).

iii) Let $y, s, t \in S$ be such that $(y s, y t) \in \rho$. Then $y s x S \cap y t x S \neq \varnothing$ for all $x \in S^{1}$ and so $s x S \cap t x S \neq \varnothing$ since $S$ is left cancellative. Hence $(s, t) \in \rho$.

Roughly speaking, $\tau$ is a measure of how far a cancellative semigroup $S$ is from being an Ore semigroup. Following the standard terminology, $[1], \S 1.10$, we call $\tau$ the reversive congruence on $S$.

Assume that there exist $0 \neq a, c \in K[S]$ such that axc $=0$ for every $x \in S^{1}$. Let $a=\lambda_{1} s_{1}+\ldots+\lambda_{n} s_{n}$ for $s_{i} \in S, \lambda_{i} \in K$, where the $s_{i}$ are distinct and the $\lambda_{i}$ are nonzero. If $\left(s_{1}, s_{2}\right) \notin \rho$, then we can choose $x_{12} \in S^{1}$ such that $s_{1} x_{12} S \cap s_{2} x_{12} S=\varnothing$. Otherwise we put $x_{12}=1$. We then define $a_{2}=a x_{12}, c_{2}=c x_{12}$ and note that $a_{2} x c_{2}=0$ for every $x \in S^{1}$. Next, we proceed in the same way with respect to the pair $t_{1}, t_{3}$ in the presentation $a_{2}=\lambda_{1} t_{1}+\ldots+\lambda_{n} t_{n}$ where $t_{i}=s_{i} x_{12}$. Repeating this for all pairs $1, j$, where $j \in$ $\{2, \ldots, n\}$, and then for all remaining pairs $i<k, i, k \in\{1, \ldots, n\}$, we come to the elements

$$
b=a x_{12} x_{13} \ldots x_{23} x_{24} \ldots x_{(n-1) n}, \quad d=c x_{12} x_{13} \ldots x_{23} x_{24} \ldots x_{(n-1) n}
$$

such that $b x d=0$ for every $x \in S^{1}$. Moreover, the following condition is satisfied for $y=x_{12} x_{13} \ldots x_{23} x_{24} \ldots x_{(n-1) n}$

$$
\text { if } s, t \in \operatorname{supp}(a) y \text { and }(s, t) \notin \rho \text {, then } s S \cap t S=\varnothing
$$

The same procedure can be then applied to the elements in $\operatorname{supp}(c) y$ (in place of the elements of $\operatorname{supp}(a)$ ). Thus, replacing $y$ by an appropriate right multiple of $y$, we can also assume that the following is true:

$$
\text { if } s, t \in \operatorname{supp}(c) y \text { and }(s, t) \notin \rho \text {, then } s S \cap t S=\varnothing
$$

A similar argument (involving $\rho^{\prime}$ in place of $\rho$, and the left multiplications by an appropriate sequence of elements of $S^{1}$ in place of the right multiplications by $\left.x_{12}, x_{13}, \ldots, x_{(n-1) n}\right)$ can be applied to the elements of $\operatorname{supp}(a) y$ and $\operatorname{supp}(c) y$ successively. This yields an element $u \in S$ such that the following condition is satisfied:

$$
\text { if } s, t \in u(\operatorname{supp}(a)) y \text { and }(s, t) \notin \rho^{\prime} \text {, then } S s \cap S t=\varnothing,
$$

and similarly

$$
\text { if } s, t \in u(\operatorname{supp}(c)) y \text { and }(s, t) \notin \rho^{\prime}, \text { then } S s \cap S t=\varnothing .
$$

More can be claimed if $S$ is left cancellative. Let $e \in\{a, c\}$ and let $z, v \in \operatorname{supp}(e)$ be such that $(u z y, u v y) \notin \rho$. Then $(z y, v y) \notin \rho$ and $(1),\left(1^{\prime}\right)$ imply that $z y S \cap v y S=\varnothing$. Since $\rho$ is left cancellative by Lemma 1 , this yields $u z y S \cap u v y S=\varnothing$. Hence, in this case, the 
following assertion is also true for $e \in\{a, c\}$ :

$$
\text { if } s, t \in u(\operatorname{supp}(e)) y \text { and }(s, t) \notin \rho, \text { then } s S \cap t S=\varnothing .
$$

To state the technical lemma below it is convenient to fix some notation. Let $a y=a_{1}+\ldots+a_{k}$ where each $a_{i}$ is of the form $\sum_{l} \lambda_{l} s_{l} y$ with the summation running over the set $A_{i} \subseteq \operatorname{supp}(a)$ such that $A_{i} y$ lies in a $\rho$-class of $S$ and $\operatorname{supp}(a) y$ is a disjoint union of $A_{1} y, \ldots, A_{k} y$. From (1) it follows that $\left(A_{i} y S\right) \cap\left(A_{j} y S\right)=\varnothing$ for $j \neq i$. Therefore, the equality $(a y) x c=0$ implies that $a_{i} x c=0$ for $i=1, \ldots, k$, and all $x \in S^{1}$.

Let $c=\mu_{1} t_{1}+\ldots+\mu_{w} t_{w}, \mu_{i} \in K, t_{i} \in S$, where the $t_{i}$ are distinct and the $\mu_{i}$ are nonzero. Then $c=c_{1}+\ldots+c_{m}$ where each $c_{i}$ is of the form $\sum_{j} \mu_{j} t_{j}$ with the summation running over the set $C_{i} \subseteq \operatorname{supp}(c)$ such that $C_{i}$ lies in a $\rho$-class of $S$ and $\operatorname{supp}(c)$ is a disjoint union of $C_{1}, \ldots, C_{m}$.

Let uay $=b_{1}+\ldots+b_{q}$ where every $b_{j}$ is of the form $\sum_{l} \lambda_{l} u s_{l} y$ with the summation running over the set $B_{j} \subseteq \operatorname{supp}(a)$ such that $u B_{j} y$ lies in a $\rho$-class of $S$ and $u(\operatorname{supp}(a)) y$ is a disjoint union of $u B_{1} y, \ldots, u B_{q} y$.

Let uay $=e_{1}+\ldots+e_{p}$ where every $e_{i}$ is of the form $\sum_{l} \lambda_{l} u s_{l} y$ with the summation running over the set $E_{i} \subseteq \operatorname{supp}(a)$ such that $u E_{i} y$ lies in a $\tau$-class of $S$ and $u(\operatorname{supp}(a)) y$ is a disjoint union of $u E_{1} y, \ldots, u E_{p} y$.

Let $u c y=d_{1}+\ldots+d_{r}$ where every $d_{j}$ is of the form $\sum_{l} \mu_{l} u t_{l} y$ with the summation running over the set $D_{j} \subseteq \operatorname{supp}(c)$ such that $u D_{j} y$ lies in a $\tau$-class of $S$ and $u(\operatorname{supp}(c)) y$ is a disjoint union of $u D_{1} y, \ldots, u D_{r} y$.

With the above notation we derive the following result.

Lemma 2. Assume that axc $=0$ for some $0 \neq a, c \in K[S]$ and all $x \in S^{1}$. Then

i) $a_{i} x c=0$ for every $x \in S^{1}, i=1, \ldots, k$.

ii) If $S$ is left cancellative, then $a_{i} x c_{j}=0$ and $b_{i} x c_{j}=0$ for every $i, j$ and all $x \in S^{1}$. Moreover $q=k$ and $b_{i}=u a_{i}$ for all $i$. for all $i, j$.

iii) If $S$ is cancellative, then $e_{i} x d_{j}=0$ for every $i, j$ and all $x \in S^{1}$. Moreover, $e_{i}, d_{j} \neq 0$

Proof. i) follows from what has been said above

ii) Assume that $S$ is left cancellative. From (3) it follows that $b_{i} x c=0$ for every $i$. If $\operatorname{sxp}=t x q$ for some $s, t \in u B_{i} y$ and some $p, q \in \operatorname{supp}(c)$, then $(\operatorname{sxp}, t x p) \in \rho$ because $(s, t) \in \rho$. Hence $(t x q, t x p) \in \rho$ and Lemma 1 iii) shows that $(p, q) \in \rho$. This easily implies that $b_{i} x c_{j}=0$ holds for every $i, j$ and all $x \in S^{1}$. The left cancellativity of $\rho$ also implies that $q=k$ and $b_{i}=u a_{i}$ for $i=1, \ldots, k$. This proves ii).

iii) The reasoning of the proof of ii) applied to the identity $a x(u c y)=0$ in place of $a x c=0$, followed by an application of the left-right dual argument involving assertions (2), $\left(2^{\prime}\right)$ can be used to establish iii).

Lemma 3. Assume that $T$ is a cancellative semigroup generated by a subset $F$ such that $F$ lies in a single $\rho$-class in $T$. Then $T$ has a group $H$ of right fractions. 
Proof. We have to show that $s T \cap t T \neq \varnothing$ for every $s, t \in T$. It is enough to check this for $s=s_{1} s_{2} \ldots s_{m}, t=t_{1} t_{2} \ldots t_{m}$ where $m \geq 1$ and $s_{i}, t_{i} \in F$. Since $\left(s_{m}, t_{m}\right) \in \rho$, there exist $u_{1}, v_{1} \in T$ such that $s_{m} u_{1}=t_{m} v_{1}$. Then $s_{1} \ldots s_{m-1} s_{m} u_{1}=s_{1} \ldots s_{m-1} t_{m} v_{1}$. Now, there exist $u_{2}, v_{2} \in T$ such that $s_{m-1}\left(t_{m} v_{1}\right) u_{2}=t_{m-1}\left(t_{m} v_{1}\right) v_{2}$ because $\left(s_{m-1}, t_{m-1}\right) \in \rho$. Hence $s_{1} \ldots s_{m-1} s_{m} u_{1} u_{2}=s_{1} \ldots s_{m-1} t_{m} v_{1} u_{2}=s_{1} \ldots s_{m-2} t_{m-1} t_{m} v_{1} v_{2}$. Proceeding this way we find elements $u_{i}, v_{i} \in T$ such that $s_{1} \ldots s_{m} u_{1} \ldots u_{m}=t_{1} \ldots t_{m} v_{1} \ldots v_{m}$. This proves the assertion.

We are now able to prove our first main result.

THEOREM 1. Let $S$ be a cancellative semigroup. Then

i) $K[S / \tau]$ is prime. In particular, $B(K[S]) \subseteq I(\tau)$.

ii) $K[S / \rho]$ is prime.

iii) If $\operatorname{ch}(K)=0$, then $K[S]$ is semiprime.

Proof. i) By Lemma 1 ii) the congruence $\tau_{S / \tau}$ is trivial. Hence, to show that $K[S / \tau]$ is prime it is enough to prove that $K[S]$ is prime whenever $\tau$ is trivial. Suppose that $K[S]$ is not prime. Then there exist $0 \neq a, c \in K[S]$ such that $a x c=0$ for all $x \in S^{1}$. Lemma 2 implies that there exist $u, y \in S$ such that $e_{i} x d_{j}=0$ for all $i, j$ where $u a y=e_{1}+\ldots+e_{p}$ and $u c y=d_{1}+\ldots+d_{r}$ for nonzero elements $e_{i}, d_{j}$ such that each $\operatorname{supp}\left(e_{i}\right)$ (and also each $\left.\operatorname{supp}\left(d_{j}\right)\right)$ is contained in a single $\tau$-class of $S$. Since $\tau$ is trivial, this implies that $e_{i}, d_{j} \in S$, a contradiction. Hence, $K[S]$ is prime.

The remaining assertion in i) follows since $I(\tau)$ is the kernel of the natural homomorphism $K[S] \rightarrow K[S / \tau]$.

ii) Since $\tau_{S / \rho} \subseteq \rho_{S / \rho}$ and the latter is trivial by Lemma 1, the assertion follows from i).

iii) Suppose that $B(K[S]) \neq 0$. Then there exists $a \in K[S], a \neq 0$ such that $a x a=0$ for every $x \in S^{1}$. Choose the minimal integer $n$ for which the following condition is satisfied: there exist a cancellative semigroup $U$ and an element $0 \neq b \in K[U]$ such that $b x b=0$ for all $x \in U^{1}$ and $|\operatorname{supp}(b)|=n$. Using Lemma 2 with respect to the semigroup $T=$ $\langle\operatorname{supp}(b)\rangle$ and the congruence $\tau_{T}$ we conclude that $\operatorname{supp}(b)$ lies in a single $\tau_{T}$-class of $T$. Therefore, by Lemma 3 , we can assume that $T=\langle\operatorname{supp}(b)\rangle$ has a group $H$ of right fractions. It is well known that $B(K[H])=0$ because $\operatorname{ch}(K)=0$. From [7], Theorem 19 in Chapter 7, it thus follows that $B(K[T])=0$. Since $b K[T] b=0$, it is clear that $b \in B(K[T])$. This contradiction completes the proof.

Remark 1. Assume that $S$ is cancellative. The proof of Theorem 1 together with the known characterizations of prime and semiprime group rings, cf. [8], provides sufficient conditions for $K[S]$ to be prime or semiprime. Namely, $K[S]$ is prime (semiprime) whenever for every subsemigroup $T$ of $S$ that has a group of fractions $H$, the group ring $K[H]$ is prime (semiprime respectively). (In the prime case, starting with an identity axc $=0$ for all $x \in S^{1}$ we can choose $z \in \operatorname{supp}(a)$ and $w \in \operatorname{supp}(c)$ and consider the identity $(w a) x(c z)=0$. Then $w z \in \operatorname{supp}(w a), \operatorname{supp}(c z)$. Choosing $a, c$ with minimal $|\operatorname{supp}(a)|+$ $|\operatorname{supp}(c)|$ we can assume, as in the proof of Theorem 1 iii), that $T=\langle\operatorname{supp}(w a), \operatorname{supp}(c z)\rangle$ has a group of right fractions $H$. Then, again by [7], Theorem 19 in Chapter 7, (wa) $x(c z)=0$ is an identity in $K[H]$.)

If $u v^{-1} \in \Delta(H)$, where $u, v \in T$, then there exists $k \geq 1$ such that $u^{k} v=v u^{k}$, so that the elements $s=u^{k}, t=v u^{k-1}$ commute and $u v^{-1}=s t^{-1}$. This leads to the following: 
i) $K[S]$ is prime whenever $S$ has no elements $s, t$ such that $s t=t s, s \neq t$, and $s^{n}=t^{n}$ for some $n>1$.

ii) if $\operatorname{ch}(K)=p>0$, then $K[S]$ is semiprime whenever $S$ has no elements $s, t$ such that $s t=t s, s \neq t$ and $s^{p}=t^{p}$.

Clearly, as seen in the group ring case, these conditions are not necessary.

2. The FC-congruence and the necessary conditions. When studying prime rings $K[S]$, as well as the semiprime case in positive characteristics, we will need a stronger congruence that naturally arises from the above considerations. It will play the role played by the FC-centre $\Delta(G)$ of a given group $G$ in the study of the group ring $K[G]$, cf. [8]. The first attempt towards a generalization of $\Delta$-methods to the case of semigroup rings of arbitrary cancellative semigroups was made by Krempa in [4], [5], where the notion of $\Delta$-subsemigroups was created. A similar idea was later developed by Dauns in $[2],[3]$.

For every cancellative semigroup $S$ a subsemigroup $\Delta(S)$ is defined by $\Delta(S)=$ $\left\{s \in S \mid\right.$ for every $x \in S$ there exists $s^{x} \in S$ such that $x s=s^{x} x$ and the set $\left\{s^{x} \mid x \in S\right\}$ is finite . It was shown in [7] that $K[\Delta(S)]$ is prime (semiprime) if $K[S]$ is prime (semiprime). (Moreover, primeness and semiprimeness of the former are characterized by conditions given in Remark 1.) Then, we asked whether the converse is true. This supposition clearly was too optimistic, even in the case where $S$ embeds into a group, as the following example shows. Let $G$ be the direct product of the free nonabelian group $H$ on $x, y$ and the group $Z_{2}=\{e, z\}$. Let $S=\langle x, y\rangle^{1} \cup\langle x, y\rangle z$. Then $K[S]$ is not prime (and it is not semiprime if $\operatorname{ch}(K)=2$ ) because $x z, x$ satisfy the condition of Theorem 2 below. On the other hand $\Delta(S)=\{1\}$, so that $K[\Delta(S)]$ is prime.

We show that an appropriately chosen congruence on $S$ should play the role played by the FC-centre in the study of prime and semiprime group rings.

Let $\omega$ be the relation defined on an arbitrary semigroup $S$ by the rule

$(s, t) \in \omega$ if there exists a finite set $F \subseteq S$ that lies in a single $\tau$-class of $S$ and such that $s x F \cap t x F \neq \varnothing$ for every $x \in S^{1}$.

We claim that $\omega$ is a congruence on $S$. Clearly, $\omega$ is reflexive and symmetric. Assume that $(s, t) \in \omega,(t, u) \in \omega$ and let $F, G$ be the suitable finite sets chosen for $s, t$ and $t, u$. If $x \in S^{\prime}$, then $s x s^{\prime}=t x t^{\prime}$ and $t\left(x t^{\prime}\right) t^{\prime \prime}=u\left(x t^{\prime}\right) u^{\prime}$ for some $s^{\prime}, t^{\prime} \in F$, and $t^{\prime \prime}, u^{\prime} \in G$. Consequently $s x s^{\prime} t^{\prime \prime}=u x t^{\prime} u^{\prime}$. Then $(s, u) \in \omega$ because $F G$ is a finite set every element of which is in the $\tau$-class of $S$ in which $s^{\prime} t$ ' lies. Let $z \in S$. It is easy to see that the set $F$ can be used to show that $(z s, z t) \in \omega$ and $(s z, t z) \in \omega$. This proves the claim. It is clear that $\omega \subseteq \rho$. We call $\omega$ the FC-congruence on $S$.

If $(s, t) \in \omega$, then by $F_{s, \ell}$ we will denote a finite subset of $S \times S$ that satisfies the following two conditions

*) there exists $u \in S$ such that for every $(c, d) \in F_{s, t}$ we have $(c, u) \in \tau,(d, u) \in \tau$.

$\left.{ }^{* *}\right) F_{s, t}$ is minimal (subject to the inclusion relation) with respect to the property that for every $x \in S^{1}$ there exists $(c, d) \in F_{s, t}$ with sxc $=t x d$.

$F_{s, t}$ need not be uniquely determined. We will often replace a given set $F_{s, t}$ by another set that satisfies $\left.\left.{ }^{*}\right),{ }^{* *}\right)$, keeping the same notation if unambiguous. 
We will further assume that $S$ is cancellative. Then, clearly $\omega$ is a left cancellative congruence and we can always choose $F_{z s, z t}=F_{s, t}$ for any given $s, t \in S, z \in S^{1}$, with $(s, t) \in \omega$.

Assume that $(s, t) \in \omega$ and that $F_{s, t}$ is chosen. Since $F_{s, t}$ is finite, there exist $n<m$, with $m \leq\left|F_{s, t}\right|+1$, such that $s s^{m} c=t s^{m} d$ and $s s^{n} c=t s^{n} d$ for some $(c, d) \in F_{s, t}$. It follows that

$$
t s^{m} d=s^{m+1} c=s^{m-n} s^{n+1} c=s^{m-n} t s^{n} d
$$

and consequently $t s^{m-n}=s^{m-n} t$ since $S$ is cancellative. Therefore, for every $s, t \in S$ with $(s, t) \in \omega$ we have $t s^{k}=s^{k} t$ for some $k \leq\left|F_{s, t}\right|$.

We are now ready to prove the following key result. Here, by $\omega^{\prime}$ we mean that left-right symmetric version of the congruence $\omega$. The finite sets chosen for every $(s, t) \in \omega^{\prime}$ will be denoted by $F^{s, t}$. Further, given $F_{s, t}$, we will always choose $F_{t, s}=$ $\left\{(d, c) \in S \times S \mid(c, d) \in F_{s, t}\right\}$. If $s x c=t x d$ for $x \in S^{1},(c, d) \in F_{s, t}$, we call the pair $(c, d)$ an $x$-conjugate of the pair $(s, t)$ in $F_{s, t}$.

Proposition. Let $S$ be a cancellative semigroup. Then

1) $\omega=\omega^{\prime}$ is a cancellative congruence on $S$.

2) For every $s, t \in S$ satisfying $(s, t) \in \omega$, the set $F_{s, t}$ can be chosen so that

i) $F_{s, t}=\left\{\left(c_{1}, d\right), \ldots,\left(c_{n}, d\right)\right\}$ for some $c_{1}, \ldots, c_{n}, d \in S$ and $\left(c_{i}, d\right) \in \omega$ for each $i$,

ii) $c d=d c$ for every $(c, d) \in F_{s, t}$,

iii) $F_{d, c}=F_{s, t}=F^{s, t}$ for every $(c, d) \in F_{s, t}$,

iv) for every $x \in S^{\prime}$ the rules $(c, d)^{x}=\left(d, c^{\prime}\right)$ if cxd $=d x c^{\prime}$ with $\left(d, c^{\prime}\right) \in F_{t, s},(d, c)_{x}=$ $\left(c^{\prime}, d\right)$ if $c^{\prime} x d=d x c$ with $\left(c^{\prime}, d\right) \in F_{s, t}$ define inverse mappings $(., .)^{x}: F_{s, t} \rightarrow F_{t, s}$ and $(., .)_{x}: F_{t, s} \rightarrow F_{s, t}$

v) for every $z \in S^{1}$ we have $F_{s, t}=F_{z s, z t}=F_{s z, z z}$.

Proof. Let $(s, t) \in \omega$. Assume that $F_{s, t}=\left\{\left(c_{1}, d_{1}\right), \ldots,\left(c_{n}, d_{n}\right)\right\}$. By the minimality of $F_{s, t}$ (see condition $\left(^{* *}\right)$ ) we know that for every $i$ there exists $x \in S^{1}$ such that $s x c_{i}=t x d_{i}$. Since $\omega$ is left cancellative and $(s, t) \in \omega$, the latter implies that $\left(c_{i}, d_{i}\right) \in \omega$ for every $i$.

By condition $\left({ }^{*}\right)$ in the definition of $F_{s, t}$ we know that $\left(d_{i}, u\right) \in \tau$ and $\left(c_{i}, u\right) \in \tau$ for some $u \in S$. In particular, there exist $w, v \in S$ such that $d_{1} w=d_{2} v$. Consider the set $F=\left\{\left(c_{1} w, d_{1} w\right),\left(c_{2} v, d_{2} v\right), \ldots,\left(c_{n} v, d_{n} v\right)\right\}$. It is clear that this set again satisfies condition $\left(^{*}\right)$. For every $x \in S^{\mathbf{l}}$ an $x$-conjugate of $(s, t)$ can be found in $F$. Since $S$ is right cancellative, it follows that $F$ also satisfies $\left(^{* *}\right)$. Therefore, replacing $F_{s, t}$ by $F$, we can assume that $d_{1}=d_{2}$. (Note that $|F|=\left|F_{s, t}\right|$ because $F_{s, t}$ satisfies $\left({ }^{* *}\right)$. In fact, if $\left(c_{1} w, d_{1} w\right)=\left(c_{i} v, d_{i} v\right)$ for some $i \geq 2$, then for any $y \in S^{1}$ the equality $s y c_{1}=t y d_{1}$ implies $s y c_{i}=t y d_{i}$, which shows that the pair $\left(c_{1}, d_{1}\right)$ could be removed from $F_{s, t}$, a contradiction). Proceeding this way with respect to the subsequent $d_{i}$ 's, we come to the case where $d_{1}=\ldots=d_{n}$, so that we can assume $F_{s, t}=\left\{\left(c_{1}, d\right), \ldots,\left(c_{n}, d\right)\right\}$. In particular, since $\left(c_{i}, d\right) \in \omega$, condition $\left.\mathrm{i}\right)$ is satisfied for $F_{s, r}$.

We know that for every $(x, y) \in \omega$ there exists $j \geq 1$ such that $x^{j} y=y x^{j}$. Then we can choose $k$ such that $d^{k} c=c d^{k}$ for each $(c, d) \in F_{s, l}$. It is clear that $s x c=t x d$ if and only if $s x c d^{k-1}=t x d^{k}$. Moreover, the elements $d^{k}, c d^{k-1}$ commute. Therefore, replacing each pair $(c, d) \in F_{s, t}$ by the pair $\left(c d^{k-1}, d^{k}\right)$, we come to a set that satisfies both $\left({ }^{*}\right)$ and $\left(^{* *}\right)$ in the definition of $F_{s, t}$, so that we can assume that $c_{i} d=d c_{i}$ for every $i=1, \ldots, n$. This proves ii). 
Let $s x c_{i}=t x d$ for some $x \in S^{1}$ and some $i$. Since $\left(c_{i}, d\right) \in \omega$, for every $y \in S^{1}$ there exist $p, q \in S$ such that $c_{i} y p=d y q$. This equality is equivalent to $s x d y q=s x c_{i} y p=t x d y p$, so that the pair $(q, p)$ can be chosen from $F_{s, t}$. It follows that $F_{c_{i}, d}$ can be chosen so that $F_{c_{i}, d} \subseteq F_{t, s}$.

Suppose now that $s x c_{i}=t x d, s x c_{k}=t x d$ for some $i, k$ and some $x \in S^{1}$. Then $i=k$, so that the $x$-conjugate of $(s, t)$ is uniquely determined in $F_{s, t}$. We denote it by $(s, t)^{x}$.

If $c_{i} z d=d z c_{j}, c_{i} z d=d z c_{k}$ for some $i, j, k$ and some $z \in S^{1}$, then $j=k$. It follows that $z$-conjugation determines a function $(., .)^{z}: F_{s, t} \rightarrow F_{t, s}$. Similarly one shows that for every $z \in S^{1}, z$-conjugation is an embedding of $F_{s, t}$ into $F_{t, s}$. Since $\left|F_{s, t}\right|=\left|F_{t, s}\right|$ is finite, it follows that $F_{s, t}^{x}=F_{t, s}$. This means that for every $\left(c_{j}, d\right) \in F_{s, t}$ and every $x \in S^{1}$ there exists $\left(c_{i}, d\right) \in F_{s, t}$ such that $d x c_{j}=c_{i} x d$. In other words, the elements $c_{j}, d$ satisfy the left-right symmetric version of the definition of $\omega$. Moreover, we get a mapping $(.,)_{x}: F_{t, s} \rightarrow F_{s, t}$ given by $\left(d, c_{j}\right)_{x}=\left(c_{i}, d\right)$. Clearly $(.,)^{x}$ and $(.,)_{x}$ are inverse functions, which establishes iv).

Let $y \in S^{1}$. Choose $x \in S^{1}$ so that $s x c_{i}=t x d$. Since we know that the $y s x$-conjugation is an onto mapping $F_{s, t} \rightarrow F_{t, s}$, it follows that there exists $j$ such that $\left(c_{j}, d\right)^{y s x}=\left(d, c_{i}\right)$. Then

$$
d y(t x d)=d y\left(s x c_{i}\right)=c_{j} y s x d
$$

and consequently $d y t=c_{j} y s$. Therefore $(s, t) \in \omega^{\prime}$ with a finite set $F^{s, t}$ chosen in $F_{s, t}$. In particular, $\omega=\omega^{\prime}$ and it is a cancellative congruence because $\omega$ is left cancellative. This completes the proof of 1 ).

We have shown that $F^{s, t} \subseteq F_{s, t}$. The left-right symmetry of $\omega$ allows us to prove that $F_{s, t} \subseteq F^{s, t}$, so that $F^{s, t}=F_{s, t}$. If $z \in S$, then we can clearly choose $F^{s z, t z}=F^{s, t}=F_{s, t}$. Since, as above, we can also have $F^{s z, t z}=F_{s z, t z}$, it follows that $F_{s z, t z}=F_{s, t}$. It is clear that we can choose $F_{z s, z t}=F_{s, t}$. This proves v).

Since $F^{s, t}=F_{s, t}$, for every $j$ there exists $y \in S^{1}$ such that $c_{j} y s=d y t$. Again, if $s x c_{i}=t x d$, it follows that

$$
c_{j} y s x d=d y t x d=d y s x c_{i}
$$

and so $\left(d, c_{j}\right)^{y s x}=\left(c_{i}, d\right)=(s, t)^{x}$. Therefore $F_{s, t} \subseteq F_{d, c_{j}}$. Since $F_{s, t}$ was chosen so that the converse inclusion is satisfied, we come to $F_{d, c_{j}}=F_{s, t}$ for every $j=1, \ldots, n$. This shows that iii) is satisfied, completing the proof of the proposition.

Remark 2. Assume that $S$ has a group $G$ of right fractions. Let $s, t \in S$ be such that $s \Delta(G)=t \Delta(G)$. Then $t s^{-1} \in \Delta(G)$ has finitely many conjugates in $G$, which therefore can be written as $c_{1} d^{-1}, \ldots, c_{n} d^{-1}$ for some $c_{i}, d \in S$. Since $c_{i} d^{-1} \in \Delta(G)$, there exists $k \geq 1$ such that $d^{k} c_{i}=c_{i} d^{k}$ for every $i$. Thus, replacing $c_{i}$ by $c_{i} d^{k-1}$ and $d$ by $d^{k}$, we can assume that $c_{i} d=d c_{i}$ for every $i$. Let $F=\left\{\left(c_{i}, d\right) \mid i=1, \ldots, n\right\}$. For every $x \in S^{1}$ there exists $i$ with $c_{i} x s=d x t$. Thus $(s, t) \in \rho^{\prime}$ and similarly $\left(c_{i}, d\right) \in \rho^{\prime}$. Clearly $\rho=S \times S$, so that $\left(c_{i}, d\right) \in \tau$ for every $i$. By the definition of $\omega^{\prime}$ it follows that $(s, t) \in \omega^{\prime}$, and so Proposition implies that $(s, t) \in \omega$. Conversely, assume that $s, t \in S$ are such that $(s, t) \in \omega$. Then $A=\left\{\left(s^{-1} t\right)^{x} \mid x \in S^{1}\right\}$ is a finite set such that $A^{y}=A$ for every $y \in S^{1}$. Consequently $A=A^{y^{-1}}$ and since $G=S S^{-1}$ (it is enough to assume that $G$ is a group generated by $S$ here), it follows that $A$ is the set of all $G$-conjugates of $s^{-1} t$. Therefore $s^{-1} t \in \Delta(G)$. This 
proves that $\omega$ coincides with the restriction to $S$ of the congruence determined on $G$ by $\Delta(G)$.

One might ask why the assumption that the $S$-conjugates of the pair $(s, t)$ can be chosen from a single $\tau$-class of $S$ is added in the definition of $\omega$. First, Remark 2 shows that $\omega$ is an extension of the notion of the FC-centre in the class of groups. Moreover, the proof of Theorem 1 shows that, whenever $a x c=0$ for some $a, c \in K[S]$ and all $x \in S^{1}$, then $a, c$ can be chosen so that $\operatorname{supp}(a), \operatorname{supp}(c)$ lie in a $\tau$-class of $S$. It is then natural to expect that a description of prime and semiprime rings $K[S]$ involves this "local" restriction on $F_{s, l}$. Finally, without this restriction, we are not able to adjust $F_{s, t}$ to a single $\omega$-class of $S$. The latter will be crucial in the proof of Theorem 2. As we have seen in Proposition, this additional condition implies that the definition of $\omega$ is left-right symmetric and consequently $\omega$ is a cancellative congruence. It is not clear whether the same can be claimed without our restriction. The problem comes from the absence of the following "equality of fractions" condition (see Malcev's condition, [1], \$ 12.4):

$$
\begin{aligned}
& \text { if } s x c=t x d, s x c^{\prime}=t x d^{\prime} \text { and } s y c=t y d \text { for some } \\
& s, t, c, d, c^{\prime}, d^{\prime}, x, y \in S \text {, then } s y c^{\prime}=t y d^{\prime} .
\end{aligned}
$$

This would be needed in the proof of Proposition if we are not able to adjust right away the pairs in $F_{s, t}$ so that they satisfy assertion 2) i).

The main advantage of having assertion 2) i) in Proposition comes from the following observation.

Lemma 4. Let $u \in S$. Then the set $T_{u}=\left\{x \in S \mid\left(x, u^{n}\right) \in \omega\right.$ for some $\left.n \geq 1\right\}$ is a subsemigroup of $S$ that satisfies the left and right Ore conditions. Moreover, if $(s, t) \in \omega$ for some $s, t \in T_{u}$, then $s^{-1} t \in \Delta\left(G_{u}\right)$, where $G_{u}$ denotes the group of fractions of $T_{u}$.

Proof. Let $x, y \in T_{u}$. Then $\left(x, u^{n}\right) \in \omega,\left(y, u^{m}\right) \in \omega$ for some $n, m \geq 1$. Clearly $\left(x y, u^{n+m}\right) \in \omega$ so that $x y \in T_{u}$. If for example $m \geqslant n$, then $\left(x u^{m-n}, y\right) \in \omega$ and we know that there exists $k$ such that $\left(x u^{m-n}\right) y^{k}=y^{k}\left(x u^{m-n}\right)$. It follows that $x T_{u} \cap y T_{u} \neq \varnothing$ because $u^{m-n} y^{k}, y^{k-1} x u^{m-n} \in T_{u}$. A symmetric argument shows that we also have $T_{u} x \cap T_{u} y \neq \varnothing$. The remaining assertion follows from Remark 2 because $(s, t) \in \omega_{T_{u}}$ whenever $s, t \in T_{u}$ are such that $(s, t) \in \omega$.

We will need certain subgroups of the above-defined groups $G_{u}$. For $u \in S$ let $D_{u}=\left\{x^{-1} y \in G_{u} \mid x, y \in T_{u},(x, y) \in \omega\right\}$ and $H_{u}=\left\{x^{-1} y \in G_{u} \mid x, y \in T_{u},(x, y) \in \omega\right.$ and $F_{x, y}$ can be chosen in $\left.T_{u} \times T_{u}\right\}$.

Lemma 5. Let $u \in S$. Then $H_{u} \subseteq D_{u}$ are subgroups of $\Delta\left(G_{u}\right)$. Moreover, every $z \in S^{1}$ acts by "conjugation" as an automorphism of $H_{u}$, that is, $\left(x^{-1} y\right)^{z}=c d^{-1}$ where $(x, y)^{z}=(c, d) \in F_{x, y} \subseteq T_{u} \times T_{u}$.

Proof. It is clear that $D_{u}, H_{u}$ are closed under taking inverses in $G_{u}$. Assume that $x^{-1} y, w^{-1} v \in D_{u}$ where $x, y, w, v \in T_{u}$ are such that $(x, y) \in \omega,(w, v) \in \omega$. From Lemma 4 it follows that $y w^{-1}=h^{-1} g$ where $g, h \in T_{u}$. Then the elements $h x, h y=g w, g v$ lie in the same $\omega$-class of $S$. Since $\left(x^{-1} y\right)\left(w^{-1} v\right)=(h x)^{-1}(g v)$, this implies that $D_{u}$ is a subsemigroup of $G_{u}$. Therefore it is a subgroup of $G_{u}$ and $D_{u} \subseteq \Delta\left(G_{u}\right)$ by Lemma 4 .

Now, we show that for every $x^{-1} y \in H_{u}$ with $(x, y) \in \omega$ and every $z \in S^{1}$ the element $\left(x^{-1} y\right)^{z}$ is well defined in $H_{u}$. Assume that $x^{-1} y=\bar{x}^{-1} \bar{y}$ for some $\bar{x}, \bar{y} \in T_{u}$ with $(\bar{x}, \bar{y}) \in \omega$ 
and $F_{\bar{x}, \bar{y}} \subseteq T_{u} \times T_{u}$. Then there exist $a, b \in T_{u}$ such that $a x=b \bar{x}$ (cf. Lemma 4) and so $a y=b \bar{y}$. Since $x z c=y z d, \bar{x} z s=\bar{y} z t$ for some $c, d, s, t \in T_{u}$, we come to $a x z c=a y z d$, $b \bar{x} z s=b \bar{y} z t$. If $c p=s q$ for some $p, q \in T_{u}$ (existing because $c, s \in T_{u}$ ), we must have $d p=t q$. This implies that $c d^{-1}=c p(d p)^{-1}=s q(t q)^{-1}=s t^{-1}$, which shows that the rule $\left(x^{-1} y\right)^{z}=c d^{-1}$ defines the $z$-conjugates of the elements of $H_{u}$. Clearly $(c, d) \in \omega$ because $x z c=y z d$ and $(x, y) \in \omega$ (note that $\omega$ is cancellative). We know that $F_{c, d}$ can be chosen so that $F_{c, d} \subseteq F_{y_{x} x}$. Since $F_{y, x} \subseteq T_{u} \times T_{u}$, it follows that $c d^{-1} \in H_{u}$.

Next, we check that $\left(x^{-1} y\right)^{z}\left(w^{-1} v\right)^{z}=\left(x^{-1} y w^{-1} v\right)^{z}$, where $(w, v) \in \omega$ and $w^{-1} v \in$ $H_{u}$. Let

$$
\text { (\#) } x z c=y z d, \quad w z \bar{c}=v z \bar{d}
$$

for some $\bar{c}, \bar{d} \in T_{u}$. If $c d^{-1} \bar{c} \bar{d}^{-1}=e f^{-1}$ and $x^{-1} y w^{-1} v=g^{-1} h$ for $e, f, g, h \in T_{u}$, then we have to show that $g z e=h z f$. Multiplying on the left both equalities in (\#) by some elements of $T_{u}$ we can assume that $x=w$ (see Lemma 4). A similar argument (involving right multiplication) allows us to assume that $d=\bar{d}$. Next, multiplying both equalities on the left by some $x^{k}$ we can assume that $x y=y x$ (since $(x, y) \in \omega$, a power of $x$ commutes with $y$ ). Similarly, we reduce to the case where $\bar{c} \bar{d}=\bar{d} \bar{c}$. Finally, we multiply both equalities on the left by $x^{r}=w^{r}$ for $r$ such that $w^{r+1}, w^{r} v$ commute. Then, replacing $x=w$ by $w^{r+1}, v$ by $w^{r} v$, and $y$ by $w^{r} y$ we can also assume that $w v=v w$ (note that $w^{r+1}=x^{r+1}$, $w^{r} y=x^{r} y$ also commute because $x y=y x$, so that the last step does not affect the foregoing simplifications). Similarly, multiplying on the right by an appropriate power of $d$, we come to the case where $c d=d c$. Now

$$
x^{-1} y w^{-1} v=x^{-1} y x^{-1} v=x^{-2} y v, \quad c d^{-1} \bar{c} \bar{d}^{-1}=c \bar{d}^{-1} \bar{c} \bar{d}^{-1}=c \bar{c} \bar{d}^{-2}
$$

so that we can choose $g=x^{2}, h=y v, e=c \bar{c}, f=\bar{d}^{2}$. Then

$$
g z e=x(x z c) \bar{c}=x(y z d) \bar{c}=x y z \bar{d} \bar{c}=y x z \bar{c} \bar{d}=y(w z \bar{c}) \bar{d}=y(v z \bar{d}) \bar{d}=h z f .
$$

This proves the claim. Since $(x, y) \in \omega, x=w$ and $(v, w) \in \omega$, we see that $(g, h) \in \omega$. We have also shown that the set $F_{g, h}$ (consisting of the appropriate pairs $(e, f)=(g, h)^{z}, z \in$ $S^{1}$ ) can be chosen in $T_{u} \times T_{u}$. Therefore $H_{u}$ is a subsemigroup, and so a subgroup, of $D_{u}$. Hence, the $z$-conjugation is an endomorphism of $H_{u}$. By the left-right symmetry of $\omega$ there is a map $q \rightarrow q_{z}, q \in H_{u}$, such that $\left(p^{z}\right)_{z}=p$ for every $p \in H_{u}$ and $\left(q_{z}\right)^{z}=q$ (namely, if $x z c=y z d$, then $\left.\left(c d^{-1}\right)_{z}=x^{-1} y\right)$. Therefore $z$ acts as an automorphism of $H_{u}$.

Our main objective is to prove the following result.

TheOREM 2. Let $S$ be a cancellative semigroup. Assume that $s, t \in S$ are such that $(s, t) \in \omega, s^{m}=t^{m}$ for some $m>1, s \neq t$, and $s t=t s$. Then $K[S]$ is not prime. Moreover, if $\operatorname{ch}(K)=p>0$ and $m=p$, then $K[S]$ is not semiprime.

Proof. Choosing $F_{s, t}$ as in Proposition, we can assume that there exists $d \in S$ such that for every $(c, e) \in F_{s, t}$ we have $(c, d) \in \omega, d=e$ and $c d=d c$. If $x \in S^{1}$ is such that $\operatorname{sxc}=t x d$, then

$$
s^{m} x c^{m}=s^{m-1} t x d c^{m-1}=t s^{m-1} x c^{m-1} d=\ldots=t^{m} x d^{m}=s^{m} x d^{m}
$$

so that $c^{m}=d^{m}$. Hence, every pair $c, d$ inherites the hypotheses on $s, t$. Fix some $(c, d) \in F_{s, t}$. By assertion iii) of the Proposition we know that $F_{d, c}=F_{s, t} \subseteq A_{d} \times A_{d}$ where 
$A_{d}$ denotes the $\omega$-class of $d$ in $S$. Now $Z=\left\{\left(\left(d^{-1} c\right)^{i}\right)^{z} \mid z \in S^{1}, i=1, \ldots, m\right\}$ is a finite subset of the group $H_{d}$ and $Z$ consists of periodic elements (see Lemma 5). Since $H_{d}$ is an FC-group, $Z$ generates a finite group $F \subseteq H_{d}$, see [8], Lemma 4.1.5. Clearly $F^{z}=F$ for every $z \in S^{1}$ and so $y F=F y$ for every $y \in T_{d}$. Then there exists $e \in T_{d}$ such that $e F=F e \subseteq T_{d}$ (a common denominator of the elements of $F$ ). Let $a=\sum_{f \in F} f$. Then $e a=a e \in K\left[T_{d}\right]$. We will show that, for every $z \in S^{1}$, the following equality holds in $K[S]$

$$
\text { (\#) }(a e) z(a e)=|F|(a e) z e
$$

Then $(a e) z[(a e)-|F| e]=0$ and clearly $a e \neq 0$ because $a \neq 0$ and $e \in S$. If $a e=|F| e$, then $|\operatorname{supp}(a e)|=1$, so that $|\operatorname{supp}(a)|=1$. Then $F$ is a trivial group, which implies that $d=c$ because $d^{-1} c \in F$. This contradicts the fact that $s \neq t$ and shows that $(a e)-|F| e \neq 0$. Therefore (\#) implies that $K[S]$ is not prime. If additionally $\operatorname{ch}(K)=m$, then $|F|=0$ in $K$ because $d^{-1} c \in F$ has order $m$. Therefore $K[S]$ is not semiprime in this case.

It remains to prove that (\#) holds. Since $e F=F e \subseteq T_{d}$, we know that $F=\left\{a_{i} e^{-1} \mid i=\right.$ $1, \ldots, n\}=\left\{e^{-1} a_{i} \mid i=1, \ldots, n\right\}$ for some $a_{i} \in T_{d}$. For every $z \in S^{1}$ the $z$-conjugation is an automorphism of $F$, so that for every $i$ there exists $j$ such that $a_{i} z e=e z a_{j}$. Moreover, $i \rightarrow j$ is a one-to-one map of the set $\{1, \ldots, n\}$ onto itself. Therefore

$$
(a e) z e=\left(\sum_{i} a_{i}\right) z e=e z\left(\sum_{j} a_{j}\right)=e z(a e) .
$$

Since this holds for every $z \in S^{1}$, we also get

$$
(a e)^{2} z e^{2}=(a e)[(a e) z e] e=e[(a e) z e](a e) .
$$

Now $a e=e a$ and $a^{2}=|F| a$ in $K\left[G_{d}\right]$, so that $(a e) e=e(a e)$ and $(a e)^{2}=|F|(a e) e$ in $K\left[G_{d}\right]$. The latter two equalities can then be viewed as equalities in $K\left[T_{d}\right] \subseteq K[S]$. Thus $|F| e(a e) z e^{2}=e(a e) z(a e) e$, and consequently $|F|(a e) z e=(a e) z(a e)$ because $e \in S$ is a regular element in $K[S]$. This proves (\#) and-completes the proof of the theorem.

Since $\omega$ plays the role that is played by the FC-centre $\Delta(G)$ in the case of a group ring $K[G]$, the necessary conditions found in Theorem 2 generalize the results known for group rings, see [8], and for semigroup rings of cancellative semigroups that have groups of fractions, [7]. We conjecture that these conditions are also sufficient.

We conclude with a few simple examples.

Example 1. Let $S$ be the Baer-Levi semigroup on a countable infinite set $A$, cf. [1]. That is, $S$ consists of all one-to-one mappings $s: A \rightarrow A$ such that $A \backslash s(A)$ is an infinite set, subject to the composition $s t=s \circ t$. It is known that $S$ is left cancellative and $S s=S$ for every $s \in S$. Hence $\rho^{\prime}=S \times S$. Let $s, t \in S$ be such that the complement of the set $Z=\{a \in A \mid s(a)=t(a)\}$ is finite. Then, for every $x \in S^{1}$ the set $X=\{a \in A \mid s x(a)=$ $t x(a)\}$ satisfies $x(X) \subseteq Z$. Moreover, $a \rightarrow x(a)$ is an injective mapping of $A \backslash X$ into $A \backslash Z$, so that $|A \backslash X| \leq|A \backslash Z|=n<\infty$. Let $A_{1}, \ldots, A_{n+1}$ be disjoint infinite subsets of $A$ such that $A=A_{1} \cup \ldots \cup A_{n+1}$. Then, for every $x \in S^{1}$, there exists $i$ such that $X \supseteq A_{i}$. Let $p_{j} \in S$ be such that $p_{j}(A) \subseteq A_{j}, j=1, \ldots, n+1$. Then $\operatorname{xp}_{i}(A) \subseteq x(X) \subseteq Z$, so that $\operatorname{sxp}_{i}=\operatorname{txp}_{i}$. It follows that $(s, t) \in \rho$, and so $(s, t) \in \tau$. Assume now that $s, t \in S$ satisfy $(s, t) \in \tau$. Suppose that the set $A \backslash Z$ is infinite. It is easy to see that there exists an infinite set $E \subseteq A \backslash Z$ such that $s(E) \cap t(E)=\varnothing$. (If $s(G) \cap t(G)=\varnothing$ for a finite set $G \subseteq A \backslash Z$, then there exists $a \in(A \backslash Z) \backslash G$ such that $s(a) \notin t(G)$ and $t(a) \notin s(G)$. Then $s(G \cup\{a\}) \cap t(G \cup\{a\})=\varnothing$, so 
that $E$ can be constructed inductively.) Let $x \in S$ be such that $x(A) \subseteq E$. Since $s x(A) \subseteq s(E)$ and $t x(A) \subseteq t(E)$, it follows that $s x S \cap t x S=\varnothing$, a contradiction. This proves that the complement of $Z$ is finite. Therefore $\tau=\rho$ is characterized by this condition. Note that $\tau$ is not cancellative. If $F_{s, t}=\left\{\left(u_{1}, v_{1}\right), \ldots,\left(u_{n}, v_{n}\right)\right\}$ is a set chosen for some $(s, t) \in \omega$, then we know that $F_{s, t} \subseteq T \times T$ for a $\tau$-class $T$ of $S$. This implies that all $u_{i}$ and $v_{i}$, viewed as functions $A \rightarrow A$, coincide on a subset $B \subseteq A$ with a finite complement. It is easy to see that this leads to $s=t$, so that the congruence $\omega$ is trivial.

We will proceed to show that $K[S]$ is prime for every field $K$. To this end we slightly modify the argument of the proof of Lemma 2. Namely, if $0 \neq a \in K[S]$ and $\operatorname{supp}(a)=$ $\left\{s_{1}, \ldots, s_{n}\right\}$, let $b_{i j} \in A, i \neq j$, be such that $s_{i}\left(b_{i j}\right) \neq s_{j}\left(b_{i j}\right)$. Set $B=\left\{b_{i j} \mid i, j=1, \ldots, n\right\}$. If $s, t \in \operatorname{supp}(a)$ are such that $(s, t) \notin \rho$, then the set $Z$ defined above has infinite complement in $A$ and as above we can find an element $x \in S$ such that $s x(A) \cap t x(A)=\varnothing$. By changing the values of $x$ at finitely many points we find a function $x^{\prime} \in S$ such that $B \subseteq x^{\prime}(A)$ and $s x^{\prime}(A) \cap t x^{\prime}(A)$ is finite. The latter implies that $s x^{\prime} S \cap t x^{\prime} S=\varnothing$, while the former ensures that $s_{i} x^{\prime} \neq s_{j} x^{\prime}$ for all $i \neq j$. Hence $a x^{\prime} \neq 0$. Thus, proceeding as in the proof of Lemma $2 \mathrm{i}$ ), we show that whenever $\operatorname{axc}=0$ for some $0 \neq c \in K[S]$ and all $x \in S^{1}$, then $a^{\prime} x c=0$ for all $x \in S^{1}$ where $a^{\prime} \neq 0$ is an element of $K[S]$ the support of which lies in a single $\tau$-class of $S$. This allows us to assume that $\operatorname{supp}(a)$ lies in a $\tau$-class of $S$. Then the set $D$ on which the functions of $\operatorname{supp}(a)$ agree has a finite complement in $A$. If $\operatorname{supp}(c)=\left\{t_{1}, \ldots, t_{m}\right\}$, then there exist $a_{i} \in A, i=2, \ldots, m$, such that $t_{1}\left(a_{i}\right) \neq t_{i}\left(a_{i}\right)$. Choose $z \in S$ such that $\left\{z t_{1}\left(a_{i}\right), z t_{i}\left(a_{i}\right) \mid i=2, \ldots, m\right\} \subseteq D$ and $z t_{1}(A) \supseteq\left\{b_{1 j} \mid j=\right.$ $2, \ldots, n\}$. If $s_{1} z t_{1}=s_{j} z t_{i}$ for some $i, j$, this implies that $(j, i)=(1,1)$. This contradicts the fact that $a z c=0$ and establishes our claim. Since this observation seems to be of independent interest, we single it out below.

THEOREM 3. Let $S$ be the Baer-Levi semigroup on a countable infinite set. Then $K[S]$ is prime for every field $K$.

EXAmple 2. Let $S$ be the semigroup determined by the generators $x_{1}, x_{2}, x_{3}, y_{1}, y_{2}, y_{3}$ subject to the relations $x_{1} y_{1}=x_{2} y_{3}, x_{1} y_{2}=x_{3} y_{1}, x_{1} y_{3}=x_{2} y_{2}, x_{3} y_{2}=x_{2} y_{1}$. It is known that $S$ is cancellative, has the unique product (u.p.) property (consequently, $K[S]$ is a domain for every field $K$ ) and does not have the two unique product (t.u.p.) property, [7]. Moreover $S$ embeds into a group, but it does not have a group of fractions. Suppose that $s, t \in S$ are such that $(s, t) \in \rho$. Assume for example that the length $|s|$ of $s$ in the generators of $S$ does not exceed $|t|$. If $y \in\left\{y_{1}, y_{2}, y_{3}\right\}$ is the terminal letter in $t$, then choose $c, d \in S$ such that $s x_{1}^{n} c=t x_{1}^{n} d$ where $n=|t|$. From the defining relations it follows that $|s|=|t|$ and further $s=t$ (see the reasoning of [7], Example 10.13). If $x \in\left\{x_{1}, x_{2}, x_{3}\right\}$ is the terminal letter of $t$, then choose $c, d \in S$ so that $s y_{1}^{n} c=t y_{1}^{n} d$. Then, a similar argument shows that $|s|=|t|$ and $s y_{1}=t y_{1}$. Therefore $s=t$. It follows that $\rho$ is the trivial congruence on $S$.

Similarly, one can show that $\rho$ is trivial in the case of the cancellative semigroup $T$ not embeddable into a group, constructed by Malcev in [6]. It is known that $T$ is a t.u.p. semigroup and that $K[T]$ is a domain.

EXAmPLE 3. Let $S$ be a cancellative monoid with $\Delta(S) \neq 1$ (for example with a nontrivial centre). Then $\omega$ is nontrivial since $(s, 1) \in \omega$ for every $s \in \Delta(S)$. In fact, for every $x \in S^{1}$ there exists $s^{x} \in S$ such that $x s=s^{x} x$ and $F=\left\{s^{x} \mid x \in S^{1}\right\}$ is a finite set. Since 
$\left(s^{x}, 1\right) \in \tau$, cf. [7], Chapter 9 , it follows that $F^{s .1}=\left\{\left(1, s^{x}\right) \mid x \in S^{1}\right\}$ can be chosen to show that $(s, 1) \in \omega^{\prime}=\omega$.

EXAMPLE 4. Let $U$ be a cancellative semigroup not embeddable into a group and let $G$ be a group with $\Delta(G) \neq 1$. Examples of semigroups not embeddable in groups and such that $\omega$ is nontrivial can be constructed by considering subsemigroups of $U \times G$.

\section{REFERENCES}

1. A. H. Clifford and G. B. Preston, Algebraic theory of semigroups, (Amer. Math. Soc., Providence, Vol. 1, 1961, Vol. 2, 1967).

2. J. Dauns, Generalized semigroup rings, Algebra Carbondale 1980, Lect. Notes in Mathematics No. 848 (Springer-Verlag 1981), 235-254.

3. J. Dauns, Centers of semigroup rings and conjugacy classes, Semigroup Forum 38 (1989), $355-364$.

4. J. Krempa, On semigroup rings, Bull. Acad. Polon. Sci. 25 (1977), 225-231.

5. J. Krempa, Special elements in semigroup rings, Bull. Acad. Polon. Sci. 28 (1980), 17-23. 686-691.

6. A. I. Malcev, On the immersion of an algebraic ring into a field. Math. Ann. 113 (1937),

7. J. Okniński, Semigroup algebras, (Marcel Dekker, 1990).

8. D. S. Passman, The algebraic structure of group rings, (Wiley, 1977).

INSTITUTE OF Mathematics

WARSAW UNIVERSITY

BANACHA 2

02-097 WARSAW 